\title{
Star Formation and the Properties of Giant Molecular Clouds in Global Simulations
}

\author{
Elizabeth J. Tasker ${ }^{1} \&$ Jonathan C. Tan ${ }^{2}$ \\ ${ }^{1}$ Department of Physics \& Astronomy, McMaster University, \\ Hamilton, ON, Canada \\ email: taskere@mcmaster.ca \\ ${ }^{2}$ Department of Astronomy, University of Florida, \\ Gainesville, FL, USA
}

\begin{abstract}
We simulated an isolated quiescent Milky Way-type galaxy with a maximum effective resolution of $7.8 \mathrm{pc}$. Clouds formed in the interstellar medium through gravitational fragmentation and became the sites for star formation. We tracked the evolution of the clouds through $300 \mathrm{Myr}$ in the presence of star formation, photoelectric heating and feedback from Type II supernovae. The cloud mass distribution agreed well with observational results. Feedback suppressed star formation but did not destroy the surrounding cloud. Collisions between clouds were found to be sufficiently frequent to be a significant factor in determining the star formation rate.
\end{abstract}

Keywords. hydrodynamics, stars: formation, ISM: clouds, Galaxy: disk, ISM: structure.

\section{Introduction}

Almost all star formation in galaxies occurs in cold, dense clumps within giant molecular clouds (GMCs), whose properties thus set the environment for the star formation process. Inevitably, GMC evolution must be a determining factor for galactic scale star formation rates.

GMCs in the Milky Way are observed to have masses up to $6 \times 10^{6} \mathrm{M}_{\odot}$, with an equally massive atomic envelope (Williams \& McKee, 1997, Blitz et al., 1990). The mass profile is well fitted by a power-law distribution:

$$
\frac{d N}{d M}=M^{-\alpha}
$$

where the exponent $\alpha$ is observed to take a value in the range $1.5-2.5$ for GMCs in the Milky Way and M33 (Rosolowsky et al., 2006).

How connected these objects are to their galactic environment is a subject of debate. It is possible that GMCs are isolated entities whose properties are independent of the galaxy's potential and each other. Alternatively, they could be coupled, leading to a link between galaxy-scale evolution on $10 \mathrm{~s} \mathrm{kpcs}$ and that of star formation on the sub-parsec scale.

Evidence that such a coupling should exist can be found in the Schmidt-Kennicutt relation (Kennicutt, 1998). This empirical law links the surface density of star formation rate to the surface density of gas:

$$
\sum_{\mathrm{sfr}} \propto \sum_{\mathrm{gas}}^{1.4 \pm 0.15}
$$



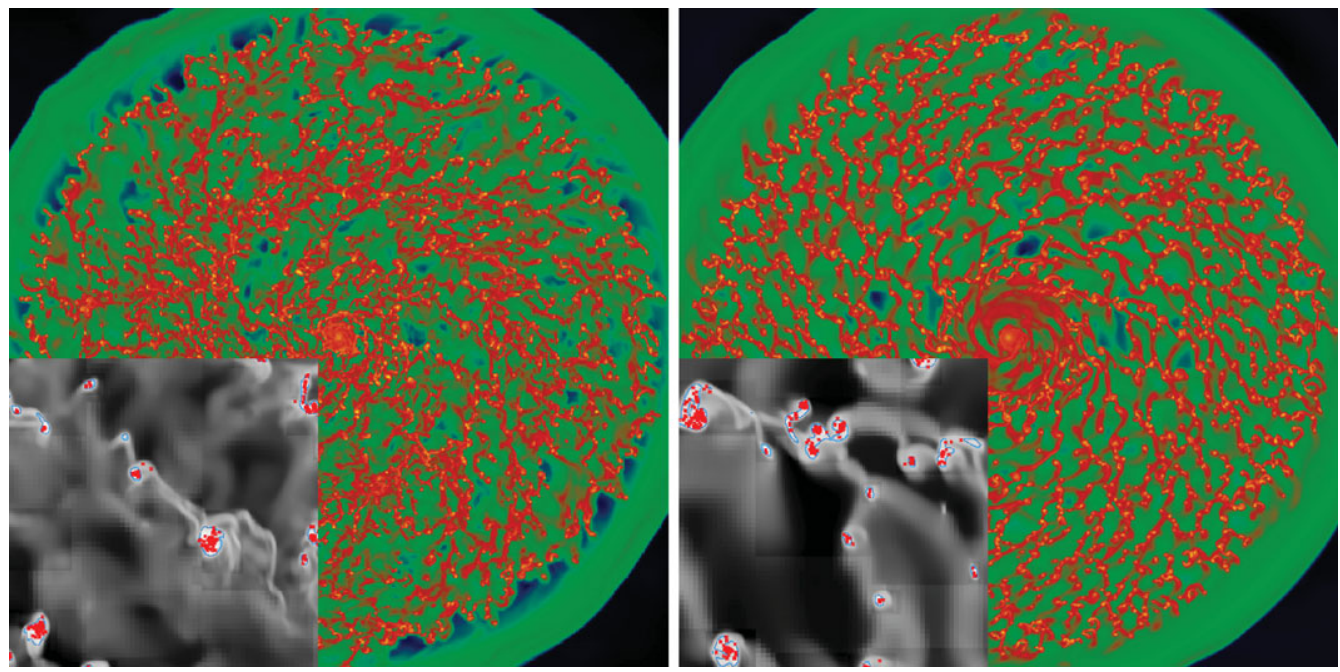

Figure 1. Surface density of the simulation with star formation (left) and the simulation with star formation and photoelectric heating. The whole disc image is $20 \mathrm{kpc}$ across, while the small cut-outs in the bottom left show a $2 \mathrm{kpc}$ slice of the density. Blue contours mark our cloud threshold density of $n_{H, c}=100 \mathrm{~cm}^{-3}$ and red points show the star particles that are less than 1 Myr old.

or alternatively, also to the orbital angular frequency at the outer radius, $\Omega_{\text {out }}: \Sigma_{\text {sfr }} \propto$ $\Sigma_{\text {gas }} \Omega_{\text {out }}$.

That the global orbital angular frequency should be related to the star formation rate suggests that star formation is strongly influenced by its large scale environment.

One method for this link is via collisions of GMCs. In his paper, Tan (2000) performed analytical calculations to show that cloud collisions could trigger star formation and produce the Schmidt-Kennicutt relation if they occurred at a frequency of a fixed fraction of their orbital period.

\section{Simulation details}

We used the adaptive mesh refinement code Enzo (Bryan \& Norman, 1997) to model the isolated galaxy disc. Details of the disc initial conditions for simulations without star formation are described in Tasker \& Tan (2009). Our highest resolution simulation has a smallest cell size of $7.8 \mathrm{pc}$ and star particles were formed with typical masses of $10^{3} \mathrm{M}_{\odot}$ from gas with $n_{H, c}>100 \mathrm{~cm}^{-3}$ (i.e. our definition of GMCs) with an efficiency of $2 \%$ per local free-fall time (Krumholz \& Tan, 2007). Radiative cooling is allowed down to $300 \mathrm{~K}$ and the gas is purely atomic, with the dense cloud gas assumed to have a high molecular component. Simulations with star formation and FUV feedback are presented here and described in more detail by Tasker \& Tan (in prep).

Clouds were defined as continuous structures with densities greater than $100 \mathrm{~cm}^{-3}$, a typical mean density of observed GMCs. They were tracked through the simulation by comparing outputs at $1 \mathrm{Myr}$ intervals. Details of the cloud identification and tracking scheme are also described in Tasker \& Tan (2009).

The surface density of the galaxy disc at $200 \mathrm{Myr}$ is shown in Figure 1. The left-hand image shows the face-on disc for a simulation that includes star formation but no form of feedback. The panel in the bottom left of the image is a $2 \mathrm{kpc}$ slice through the disc midplane. The blue contours mark out the $100 \mathrm{~cm}^{-3}$ cloud threshold and the red particles mark the stars born in the last 1 Myr. As expected, the stars are born in the identified 


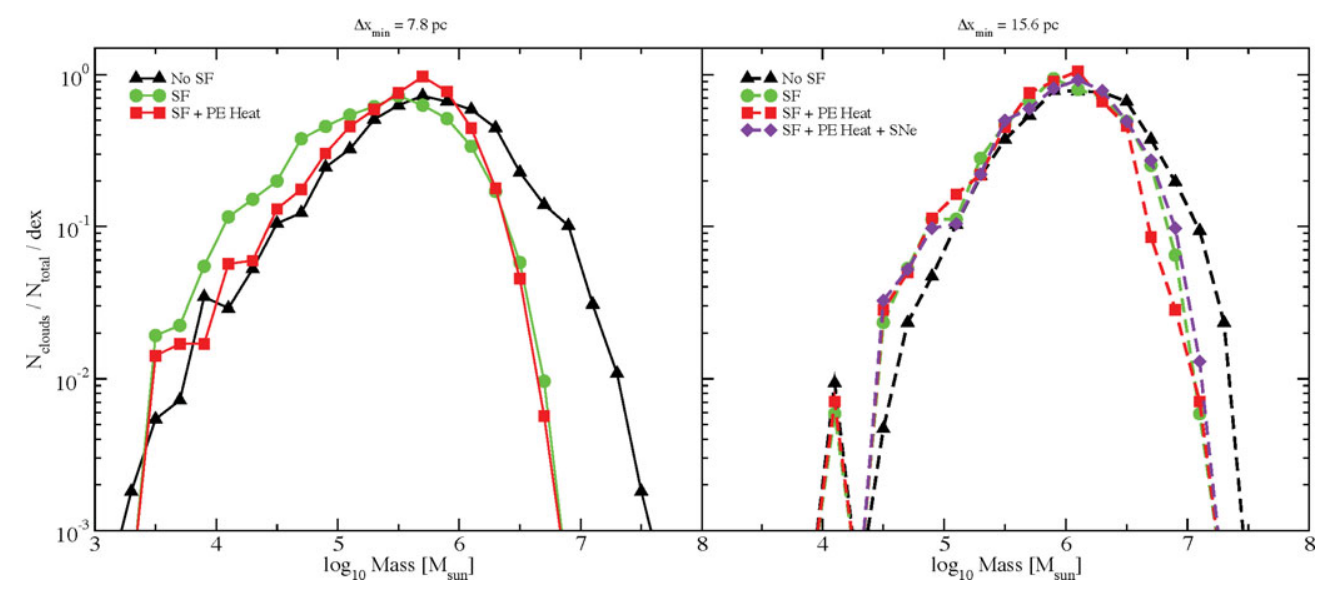

Figure 2. Distributions of GMC masses. Left-hand plot shows the results from the highest resolution $(7.8 \mathrm{pc})$ simulations with and without star formation and with the inclusion of photoelectric heating. The right plot shows the same profile for a resolution of $15.6 \mathrm{pc}$ and also includes results from the run with supernovae feedback.

GMCs. The right-hand side of Figure 1 shows the same images for the simulation with feedback from photoelectric heating. Smaller scale structures appear to be suppressed by the presence of FUV heating.

\section{Cloud properties}

Figure 2 shows the distributions of GMC masses. The left-hand plot shows clouds in the simulations with $7.8 \mathrm{pc}$ resolution. Without star formation, there is no destructive mechanism and the clouds can reach artificially large masses. With star formation, this mass reaches a maximum of $M \sim 10^{6.7} \mathrm{M}_{\odot}$. This value is unaffected by the presence of photoelectric heating. Observed GMCs in the Milky Way have masses up to $6 \times 10^{6} \mathrm{M}_{\odot}$ (Williams \& McKee, 1997) which, allowing for an atomic envelope of roughly equal size, is in good agreement with what we find.

The right-hand plot shows the mass profile at a lower resolution of $15.6 \mathrm{pc}$ and includes the simulation with supernovae feedback. We can see that the shape of the mass profile is relatively independent of the physics included. Supernovae feedback acts to greatly suppress star formation, but the relatively constant profile shape suggests that supernova feedback has only a modest influence on GMC properties.

\section{Star formation}

As mentioned in $\S 1$, cloud collision could potentially play a significant role in driving star formation. The left-hand plot in Figure 3 shows the average collision time of clouds as a fraction of their orbital time plotted against their radial position in the disc. Regardless of the physics included, the cloud collision rate is small and approximately constant fraction of the orbital time, with clouds typically experiencing a collision every quarter of an orbit. This may be sufficient for such interactions to be important for global star formation rates, if GMCs have lifetimes of this order (Tan, 2000).

The right-hand image of Figure 3 shows the Schmidt-Kennicutt relation. Squares show the gas surface density versus surface density of star formation rate averaged over disc annuli. Open circles show this relation for the individual GMCs. Over-plotted are the results from the THINGS survey (Bigiel et al., 2008) for local galaxies. Diagonal lines mark lines mark constant star formation efficiency, denoting the rate of surface star 

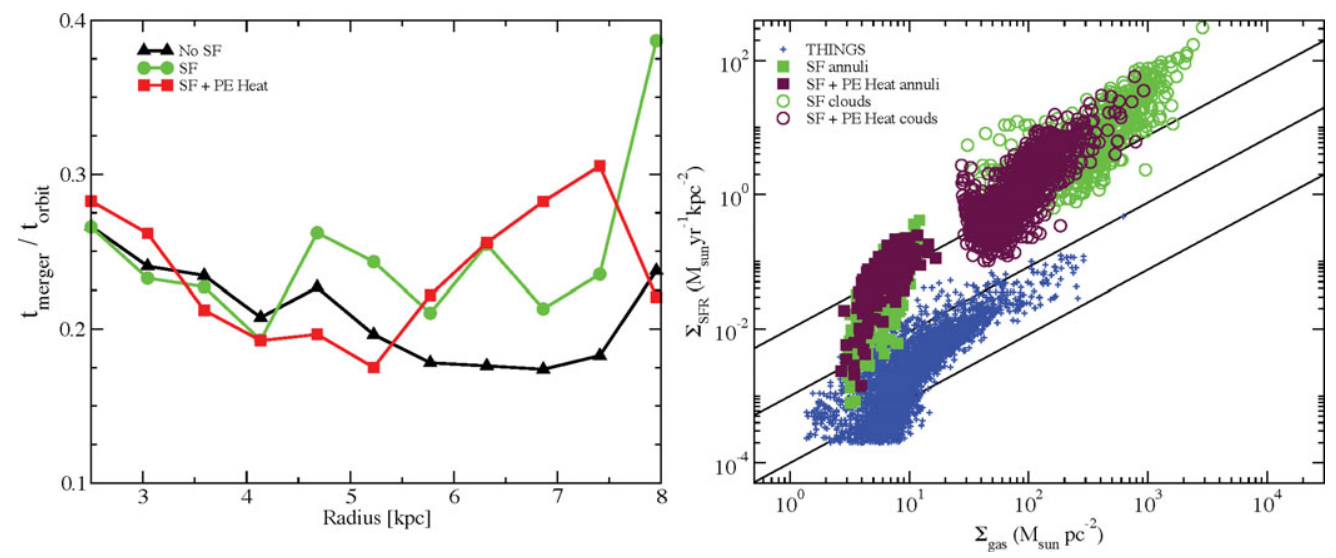

Figure 3. Left: average cloud collision rate as a function of radius for runs at $7.8 \mathrm{pc}$ resolution. Clouds from simulation times $200 \pm 25 \mathrm{Myr}$ were included. Right: the local Schmidt-Kennicutt relation for annuli in the disc and individual GMCs at 250 Myr. Solid lines mark constant star formation efficiencies and observational data from the THINGS survey is shown.

formation needed to consume 1\%, 10\% and 100\% of the gas in $100 \mathrm{Myr}$. Averaging on scales of the disk annuli, the simulations form stars at a rate about a factor of 10 higher than the observed values for a given $\Sigma_{\text {gas }}$. This is likely due to other feedback processes, such as ionization, and magnetic fields which are not yet included in our simulations.

Averaging the Schmidt-Kennicutt relation on the scale of GMCs, we find an approximate power law behavior, similar to 1.2 but with a higher normalisation. There is some evidence that on GMC scales the star formation law does have such an elevated normalization (Heiderman, Evans \& Huard, in prep.). This could be accounted for both by the physics we are not including and by the observations being averaged over a larger surface area than the individual clouds in our simulation.

\section{Summary}

We modeled isolated galaxy discs as gas orbiting in fixed axisymmetric potentials, investigating GMC formation and evolution and its dependence on star formation and feedback from diffuse background photoelectric heating and localized supernovae. Models including star formation produce GMC mass functions similar to those observed in the Milky Way, including a truncation at masses $\sim 10^{7} \mathrm{M}_{\odot}$. We find cloud collisions occur approximately every $1 / 4$ of an orbital period, indicating that this process could be a link between the global and local scales of star formation.

\section{References}

Bigiel, F., Leroy, A., Walter, F., Brinks, E., de Blok, W. J. G., Madore, B., \& Thornley, M. D. 2008, AJ, 136, 2846

Blitz, L., Bazell, D., \& Desert, F. X. 1990, ApJL, 352, L13

Bryan, G. L. \& Norman, M. L. 1997, ASP Conf. Ser. 123: Computational Astrophysics; 12th

Kingston Meeting on Theoretical Astrophysics, 363

Kennicutt, R. C., Jr. 1998, ApJ, 498, 541

Krumholz, M. R. \& Tan, J. C. 2007, ApJ, 654, 304

Rosolowsky, E., Engargiola, G., Plambeck, R., \& Blitz, L. 2003, ApJ, 599, 258

Tan, J. C. 2000, ApJ, 536, 173

Tasker, E. J. \& Tan, J. C. 2009, ApJ, 700, 358

Williams, J. P. \& McKee, C. F. 1997, ApJ, 476, 166 